\title{
Supervised Learning Technique for Geomechanical Forecasting during Underground Construction
}

\author{
Matías Lazcano', Patricio Cofré2 \\ ${ }^{1}$ SKAVA Consulting \\ Matilde Salamanca 736-601, Santiago, Chile \\ Matias.lazcano@skava.cl \\ ${ }^{2}$ MetricArts \\ Nueva Tajamar 555-1501, Santiago, Chile \\ Pcofre@metricarts.com
}

\section{Extended Abstract}

Geotechnics and geomechanics are very unpredictable sciences. Big efforts are usually made to understand rock behaviour in advance of construction in order to design, plan and quote underground projects. No matter how much is spent on exploration, it is always possible to encounter unexpected conditions during construction and be exposed to undesirable events.

This is why Geological Engineers and Geotechnics evaluate rock conditions every blast, performing a rock mass description and a rock mass classification, which helps to characterize stability and risks over the exposed area. However, no person can see behind the face. To mitigate this, mainly three methods are often used.

Core drilling can be precise, but expensive and slow [1]. Only specific conditions will justify the investment, because the face has to be stopped to perform the task. If it is done in a parallel manner, then the advance rate of the face will probably be higher than core drilling, returning useless information.

Measurement while drilling is a very powerful tool. The interpretation of digital drilling parameters can give useful information about what lies behind the face, but the investment in computerized equipment, and training of personnel imposes a huge constraint [2].

Probe holes, on the other hand, are cheap, fast and easy to perform [3]. The problem is that interpretation of data is very subjective, generally leaving the responsibility in the hands of the operator. The analysis will vary depending on which shift, equipment and operator is drilling.

Today, new techniques give new opportunities to extract knowledge from information and data available. Supervised Learning is the machine learning task of inferring a function from labelled training data [4]. The objective of this project was to apply Supervised Learning to geotechnical information, in order to find patterns and infer functions based on training data. The chosen technique was Support Vector Machine [5], and the data from Geotechnical Mapping and Probe Holes drilling was used for training.

Information from both sources had to be standardized, labelled, organized and stored in a way to be easily accessed by the supervised learning method. The model only learns from that training data, it is understood that non-experienced situations cannot be predicted. Because of this, it was assumed that every project would need its own training process.

The testing tunnel was located mainly in a Granodiorite region from Superior Cretaceous-Tertiary, with a Rhyolite unit from Cretaceous-Tertiary, and some presence of Dacite. Geomechanical stability was structurally dominated, with a periodical appearance of two fault systems. Water and stresses were not dominant factors.

1692 registers were used to train the model, and 423 registers were used as test set, all from the same tunnel. Once the model was trained, it was used as a forecasting tool during the performance of new Probe Holes. Results show that the model has an accuracy of $+85 \%$ forecasting rock mass classification behind the face in a way that is suitable for advising a decision maker. 
Surprisingly, preliminary results shown that applying the trained model in a new project also structurally dominated, can reach an accuracy of $+80 \%$ forecasting with same criteria. It is predicted that as soon as the model receives information from different scenarios, then the response for different projects will improve.

\section{References}

[1] R. E. Chapman, Petroleum Geology, Developments in Petroleum Science: ELSEVIER, 2000.

[2] D. Rivera, Aplicación de la tecnología Measurement While Drilling en Túneles, Universidad de Chile Repository, 2012.

[3] N. Bilgin and U. Ates, "Probe Drilling Ahead of Two TBMs in Difficult Ground Conditions in Turkey," Rock Mechanics Rock Engineering, 2016, vol. 49.

[4] M. Mohri, A. Rostamizadeh and A. Talwalkar, Foundations of Machine Learning, Cambridge, MA: MIT Press, 2012.

[5] S. B. Kotsiantis, Supervised Machine Learning: A Review of Classification Techniques, University of Peloponnese, 2007. 